\title{
The epidemiology of blood transfusion in hospitalized children: a national cross-sectional study in China
}

\author{
Kai Guo ${ }^{1}$, Xin-Yu Wang ${ }^{2}$, Guo-Shuang Feng ${ }^{2}$, Jian Tian ${ }^{3}$, Yue-Ping Zeng ${ }^{4}$, Shu-Xuan $\mathrm{Ma}^{1}$, Xin $\mathrm{Ni}^{2,5}$; \\ Futang Research Center of Pediatric Development (FRCPD) ${ }^{6-44}$
}

${ }^{1}$ Department of Transfusion Medicine, Beijing Children's Hospital, Capital Medical University, National Center for Children's Health, Beijing, China; ${ }^{2}$ Big Data and Engineering Research Center, Beijing Children's Hospital, Capital Medical University, National Center for Children's Health, Beijing, China; ${ }^{3}$ Hospital Administration Office, Beijing Children's Hospital, Capital Medical University, National Center for Children's Health, Beijing, China; ${ }^{4}$ Department of Medical Record Management Office, Beijing Children's Hospital, Capital Medical University, National Center for Children's Health, Beijing, China; '5epartment of Otolaryngology, Head and Surgery, Beijing Children's Hospital, Capital Medical University, National Center for Children's Health, Beijing, China; ${ }^{6}$ Medical Record Department, Dalian Children's Hospital of Dalian Medical University, Dalian, China; ${ }^{7}$ Information Department, Dalian Children's Hospital of Dalian Medical University, Dalian, China; ${ }^{8}$ Medical Record Department, Children's Hospital of Hebei Province, Shijiazhuang, China; 'Information Department, Children's Hospital of Hebei Province, Shijiazhuang, China; ${ }^{10}$ Medical Record Department, Inner Mongolia Autonomous Region Maternal and Child Health Hospital, Hohhot, China; ${ }^{11}$ Information Department, Inner Mongolia Autonomous Region Maternal and Child Health Hospital, Hohhot, China; ${ }^{12}$ Medical Record Department, Shanxi Children's Hospital, Shanxi Maternal and Child Health Care Hospital, Taiyuan, China; ${ }^{13}$ Information Center, Shanxi Children's Hospital, Shanxi Maternal and Child Health Care Hospital, Taiyuan, China; ${ }^{14}$ Medical Record Department, Anhui Children's Hospital, Hefei, China; ${ }^{15}$ Information Center, Anhui Children's Hospital, Hefei, China; ${ }^{16}$ Information Department, Hangzhou Children's Hospital, Hangzhou, China; ${ }^{17}$ Medical Record Department, Jinan Children's Hospital, Jinan, China; ${ }^{18}$ Information Department, Jinan Children's Hospital, Jinan, China; ${ }^{19}$ Medical Record Department, The Affiliated Children's Hospital of Nanchang University, Jiangxi Provincial Children's Hospital, Nanchang, China; ${ }^{20}$ Information Department, The Affiliated Children's Hospital of Nanchang University, Jiangxi Provincial Children's Hospital, Nanchang, China; ${ }^{21}$ Medical Record Department, Liaocheng Children's Hospital, Liaocheng, China; ${ }^{22}$ Information Department, Liaocheng Children's Hospital, Liaocheng, China; ${ }^{23}$ Medical Record Department, Nanjing Children's Hospital Affiliated to Nanjing Medical University, Nanjing, China; ${ }^{24}$ Information Department, Nanjing Children's Hospital Affiliated to Nanjing Medical University, Nanjing, China; ${ }^{25}$ Medical Record Department, Liuzhou Maternity and Child Healthcare Hospital, Liuzhou, China; ${ }^{26}$ Information Department, Liuzhou Maternity and Child Healthcare Hospital, Liuzhou, China; ${ }^{27}$ Medical Record Department, Shenzhen Children's Hospital, Shenzhen, China; ${ }^{28}$ Information Department, Shenzhen Children's Hospital, Shenzhen, China; ${ }^{29}$ Medical Record Department, Wuhan Children's Hospital (Wuhan Maternal and Child Healthcare Hospital), Tongji Medical College, Huazhong University of Science and Technology, Wuhan, China; ${ }^{30}$ Information Center, Wuhan Children's Hospital (Wuhan Maternal and Child Healthcare Hospital), Tongji Medical College, Huazhong University of Science and Technology, Wuhan, China; ${ }^{31}$ Information Center, Hunan Children's Hospital, Changsha, China; ${ }^{32}$ Medical Record Department, Henan Children's Hospital, Zhengzhou Children's Hospital, Children's Hospital Affiliated to Zhengzhou University, Zhengzhou, China; ${ }^{33}$ Information Department, Henan Children's Hospital, Zhengzhou Children's Hospital, Children's Hospital Affiliated to Zhengzhou University, Zhengzhou, China; ${ }^{34}$ Medical Record Department, Gansu Provincial Maternity and Child Care Hospital, Lanzhou, China; ${ }^{35}$ Medical Record Department, Women and Children's Hospital of Qinghai Province, Xining, China; ${ }^{36}$ Information Department, Women and Children's Hospital of Qinghai Province, Xining, China; ${ }^{37}$ Department of Quality Control, Women and Children's Hospital of Qinghai Province, Xining, China; ${ }^{38}$ Medical Record Department, Urumqi Children's Hospital, Urumqi, China; ${ }^{39}$ Medical Record Department, The Children's Hospital of Xi'an City, Xi'an, China; ${ }^{40}$ Information Department, The Children's Hospital of Xi'an City, Xi'an, China; ${ }^{41}$ Medical Record Department, Guiyang Children's Hospital, Guiyang, China; ${ }^{42}$ Information Center, Guiyang Children's Hospital, Guiyang, China; ${ }^{43}$ Medical Record Department, Kunming Children's Hospital, Kunming, China; ${ }^{44}$ Information Department, Kunming Children's Hospital, Kunming, China

Correspondence to: Xin Ni. Big Data and Engineering Research Center, Beijing Children's Hospital, Capital Medical University, National Center for Children's Health, No.56 Nan Lishi Rd., Xi Cheng District, Beijing, 100045, China. Email: nixin@bch.com.cn; Shu-Xuan Ma. Department of Transfusion Medicine, Beijing Children's Hospital, Capital Medical University, National Center for Children's Health, No.56 Nan Lishi Rd., Xi Cheng District, Beijing, 100045, China. Email: masxfwyy@sina.com.

Submitted Dec 18, 2020. Accepted for publication Mar 05, 2021.

doi: $10.21037 /$ tp-20-464

View this article at: http://dx.doi.org/10.21037/tp-20-464 
Children differ from adults during their growth and development. However, today, targeting specific age groups in human studies remains rare (1). Despite efforts in considering the special needs of pediatric patients and improving their targeting efficiency, therapeutic guidelines and consensuses are largely extrapolated from adult studies and reports (2). According to the study of the Canadian Laboratory Initiative on Pediatric Reference Intervals, children have a different biochemical profile than adults, except for bicarbonate. Therefore, more researches and studies are necessary to reveal the unique characteristics of the pediatric patient population.

Transfusions of red blood cells (RBCs), platelets, and plasma (3) are essential for patients with life-threatening conditions, is one of the most frequently used and critical therapies and is also associated with risks, such as transfusion-related acute lung injury, which is the onset of respiratory distress and acute lung injury due to blood product transfusion (4). Currently, the effect of the advocated restrictive transfusion strategy is still not yet completely understood (5). Among the pediatric inpatient population, the indications for blood transfusion and associated practices vary tremendously based on a variety of factors, notably, age and disease type and severity (6). Currently, some countries, such as the United Kingdom, the United States and Italy, etc., have developed guidelines or consensus recommendations on transfusions for fetuses, neonates or children $(2,7,8)$. However, these transfusion recommendations are inconsistent, and there is also an immense heterogeneity in transfusion practice. In another word, the current blood transfusion guidelines for pediatric patients are still in contention. Understanding the epidemiology of blood transfusion in clinical practice is essential to standardizing transfusion decisions. However, references for the epidemiology of blood transfusion in children are lacking. Especially in China, which is the world's most populous country, the epidemiology of blood transfusion in hospitalized children has not been well characterized. Considering the large population, there is a lack of studies or reports on the prevalence and disease spectrum of blood transfusion recipients in pediatric inpatient population to assist with formulating the national blood transfusion guidelines for children. Currently, the strategies for transfusion therapy of Chinese pediatric patients in the clinical practice are mainly dependent on blood transfusion guidelines from other countries and adults and are made on an empirical basis.

This report described and analyzed epidemiological characteristics of blood transfusion in hospitalized children based on a large, nationally pediatric patient database in China. The data on blood transfusion of pediatric patients obtained may provide a foundation for the development of evidence-based blood transfusion strategies for pediatric patients.

The Futang Research Center of Pediatric Development (FRCPD) is the first nonprofit social service organization engaging in pediatric development research in China under the supervision and management of the Ministry of Civil Affairs of China and commits itself to establish the service platform of "Care for Children in China" (http://www. futang.org/about/fu-tang-jie-shao.htm). The FRCPD includes 32 provincial and municipal medical institutions, forming an extensive pediatric diagnosis and treatment system. This study selected inpatients' medical records home page data from FRCDP's 22 tertiary children's hospitals covering the period from January 1, 2016, to December 31, 2018, and extracted information including patient name and disease code, age, sex, primary diagnosis, length of stay, and treatment information related to allogenic blood transfusion. In the study, 1 unit of blood was defined as blood products from $200 \mathrm{~mL}$ whole blood, including RBCs, plasma or platelets; 1 unit of plasma = $100 \mathrm{~mL}$ plasma, and 1 unit of platelet treatment $=10$ units of platelets. Medical records were excluded if they met any of the following conditions: (I) The age of the patient at diagnosis was older than 14 years; (II) The length of hospital stay was 0 days; (III) Key data such as patient sex, age, diagnosis or blood transfusion records were missing. Eventually, the home page data of the medical records of 3,076,096 hospitalized patients were collected. A total of 190,559 patients were treated with allogenic blood transfusion. That was, among all patients, 190,559 patients received at least 0.5 units of RBCs, 0.5 units of plasma or 0.5 units of platelet treatment transfusion during their hospitalization. The cohort characteristics of the children are presented in Table S1.

The epidemiology of blood transfusion was reported for the whole cohort and also stratified by sex, age group, and geographic region. The hospitalized patients were divided into five groups based on their age: 0-28 days (neonate); 29 days to $<1$ year (infant); 1-3 years (toddler); 4-6 years (preschool child); and 7-14 years (school-age child). The 22 children's hospitals included in our study were grouped according to the seven major geographical regions in China, namely, among Northeast China, North China, East China, South China, Central China, Northwest China and 
Southwest China.

The Tenth Revision of International Statistical Classification of Diseases and Related Health Problems (World Health Organization, ICD-10) codes (https:// www.cdc.gov/nchs/data/dvs/2e_volume1_2013.pdf), were used as the selection criteria for the primary screening and classification of disease. The primary outcome of each inpatient was determined as having at least one of the following ICD-10 procedure codes. All statistical analyses were performed using SAS JMP Pro to version 15.1 (SAS Institute, Cary, NC, USA). The continuous variables were displayed as mean \pm standard deviation $\left(\bar{x}_{ \pm} \mathrm{SD}\right)$.
The categorical variables were described as frequencies (percentages).

In this study, the total number of discharged pediatric patients nationwide was 3,076,096. The number of pediatric patients who received blood transfusion was 190,559 , with an overall prevalence of transfusions of 6.19\% (95\% CI: 6.17-6.22\%), which was higher than the pediatric transfusions prevalence in the United States $(1.07-1.59 \%)$ based on a nationally representative study reported by Goel et al. (9). The prevalence of transfusions in hospitalized children from FRCDP's 22 tertiary children's hospitals varied between $0.33 \%$ and $26.89 \%$

Table 1 The prevalent characteristics of blood transfusion during pediatric inpatient hospitalizations from 2016-2018

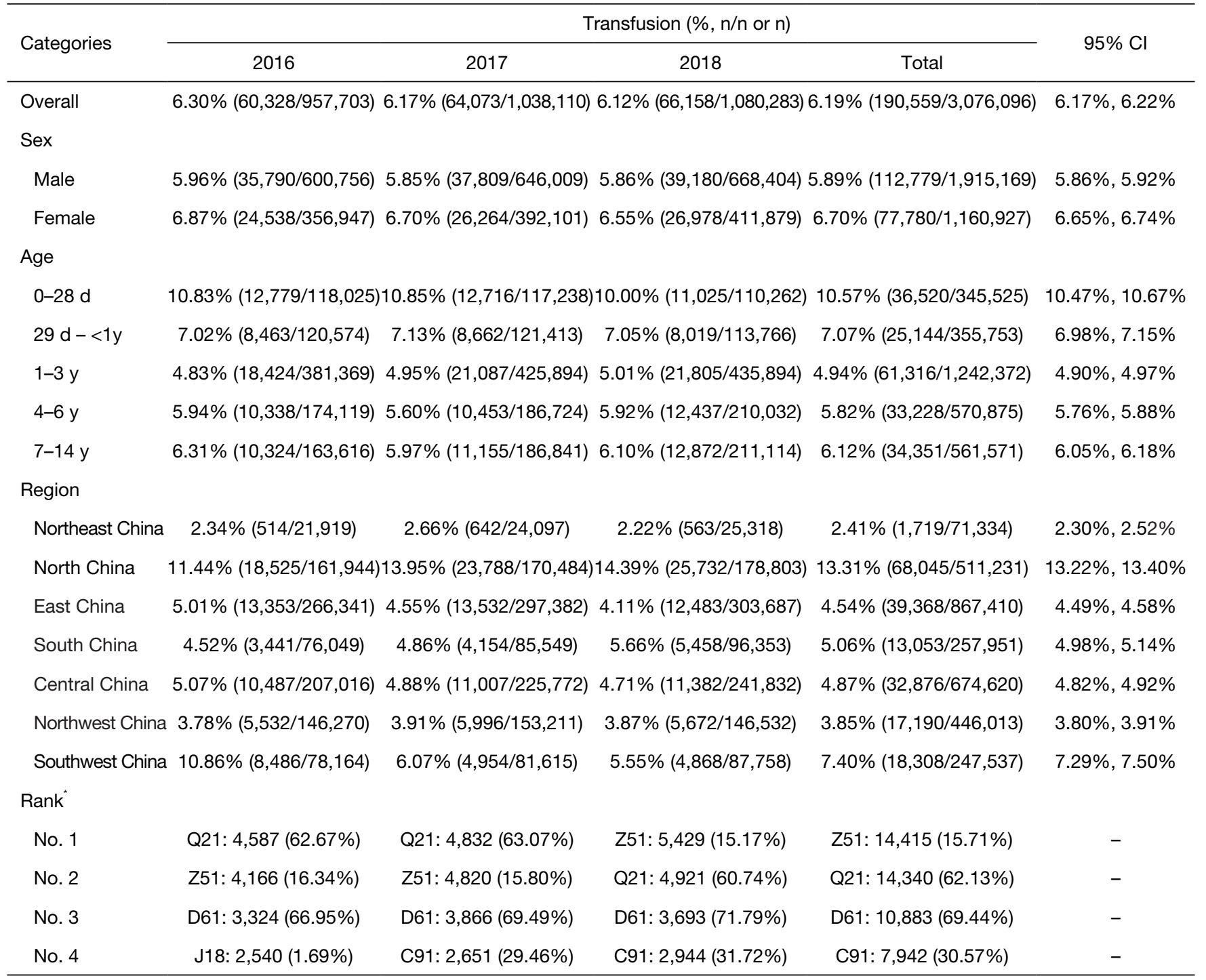

Table 1 (continued) 
Table 1 (continued)

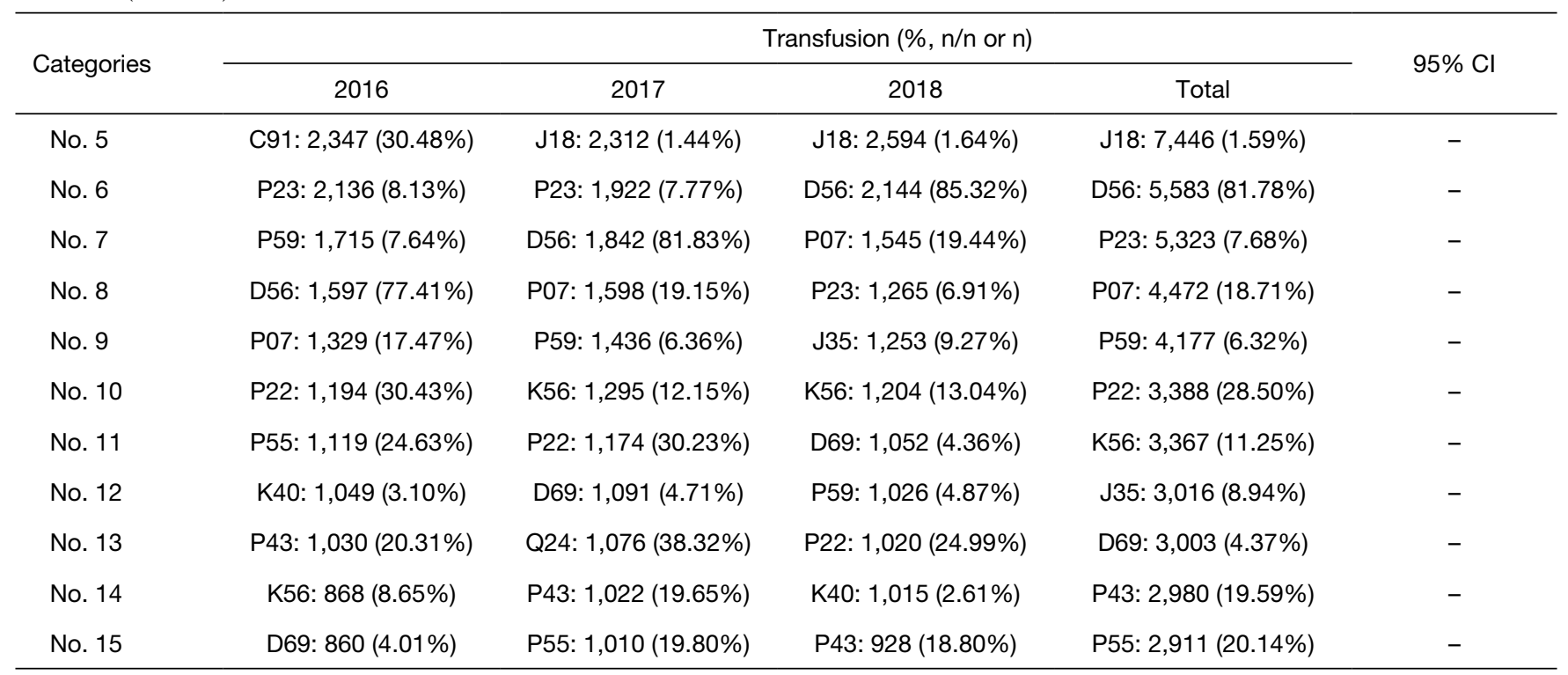

*, the disease spectrum of blood transfusion was described and ranked by calculating the total numbers of patients with the different types of diseases based on ICD-10 coding. ICD-10: Z51 (Other medical care), Q21 (Congenital malformations of cardiac septa), D61 (Other aplastic anemias), C91 (Lymphoid leukemia), J18 (Pneumonia, organism unspecified), D56 (Thalassemia), P23 (Congenital pneumonia), P07 (Disorders related to short gestation and low birth weight, not elsewhere classified), P59 (Neonatal jaundice from other and unspecified causes), P22 (Respiratory distress of newborn), K56 (Paralytic ileus and intestinal obstruction without hernia), J35 (Chronic diseases of tonsils and adenoids), D69 (Purpura and other hemorrhagic conditions), Q43 (Other congenital malformations of intestine), P55 (Hemolytic disease of fetus and newborn). K40 (Inguinal hernia), Q24 (Other congenital malformations of heart). Z51: Z51.0 (Radiotherapy session), Z51.1 (Chemotherapy session for neoplasm), Z51.2 (Other chemotherapy, Maintenance chemotherapy NOS), Z51.3 (Blood transfusion (without reported diagnosis)), Z51.4 (Preparatory care for subsequent treatment, not elsewhere classified), Z51.5 (Palliative care), Z51.6 (Desensitization to allergens), Z51.8 (Other specified medical care), Z51.9 (Medical care, unspecified). ICD, The Tenth Revision of International Statistical Classification of Diseases and Related Health Problems. CI, Confidence Interval.

(Table S2). The epidemiological characteristics of pediatric inpatients treated with blood transfusions from 2016-2018 are summarized in Table 1. More blood transfusions were performed in males [112,779; $5.89 \%$ (95\% CI: 5.86-5.92\%)] than in females [77,780; 6.70\% (95\% CI: $6.65-6.74 \%)]$, in 2016, 2017 and 2018. The majority of blood transfusion therapies were in hospitalized children that were 0-28 days old [10.57\% (95\% CI: $10.47-10.67 \%)]$, which was lower than the neonatal transfusion rate in China reported by Sun et al. (13.02\%) (10). This may be due to the differences in the pediatric sample population. The lowest prevalence of transfusions was in the group of children 1-3 years of age [4.94\% (95\% CI: 4.90-4.97\%)].

Subsequently, this study also revealed that the prevalence of transfusions in hospitalized children varies in different regions of China. The highest prevalence of transfusions was in North China [13.31\% (95\% CI: $13.22-13.40 \%$ )], whereas the lowest prevalence of transfusions was in
Northeast China [2.41\% (95\% CI: $2.30-2.52 \%)]$. The prevalence of transfusions in other regions was between $3.85 \%$ and $7.40 \%$. Moreover, while most regions maintained a fairly stable prevalence of transfusions, North China seemed to gradually but significantly increase its blood use over the three-year period studied. One explanation could be that there are more critically ill children in the pediatric hospitals of North China; for example, the representative hospital of Beijing Children's Hospital, Capital Medical University, is one of the largest general pediatric hospitals in China, with a transfusion prevalence of $9.84 \%$. It is also possible that blood availability is a factor, with blood being less available in Northeast China, for instance, compared to North China. More reasons for this increase need to be further studied. More importantly, there may be some differences in blood transfusion strategies, such as blood transfusion thresholds and evaluations of curative effects after blood transfusion, in hospitalized children of 


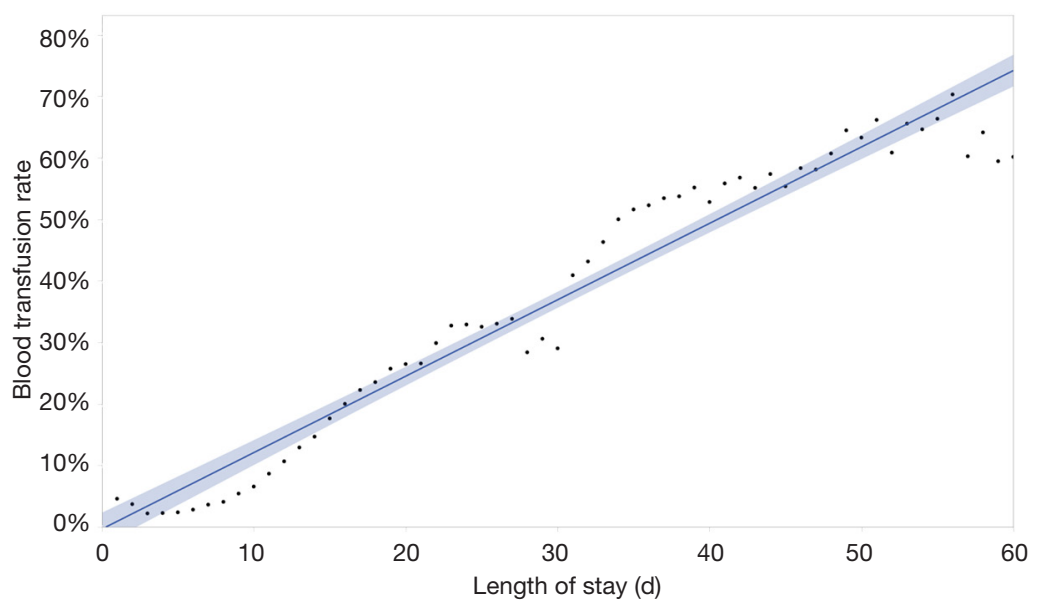

Figure 1 Association of prevalence of transfusions with length of stay in hospitalized children. R2=0.949; $\mathrm{Y}=-0.003209+0.01242$ * $\mathrm{X}, \mathrm{F}(1$, 58) $=1,083.74, \mathrm{P}<0.001$.

different sexes, ages and regions. In summary, sex, age and regional characteristics related to blood transfusion should be considered and investigated when formulating blood transfusion guidelines for the pediatric inpatient population, particularly in China.

In addition, the association of the prevalence of transfusions and the length of hospitalization was analyzed by Pearson correlation analysis. Among 3,076,096 total pediatric hospitalizations, 6,395 inpatient hospitalizations were excluded because the length of hospital stay exceeded 60 days. Nevertheless, the prevalence of transfusions was elevated along with an increase in the length of hospitalization (R2=0.949, Figure 1). One explanation could be that the length of hospitalization is related to more critical illness, blood system diseases, coagulation disorders, anemia, etc.

Furthermore, we analyzed the disease rank of blood transfusion for these 190,559 pediatric patients according to the total numbers of patients with the different types of diseases based on ICD-10 coding. This information is essential to assist the formulation of transfusion guidelines regarding the understanding of the disease spectrum in pediatric patients treated with blood transfusion; however, there are few related either inside or outside of China. The top fifteen diseases in hospitalized children treated with blood transfusion are shown in Table 1. The ICD-10 codes of the overall top fifteen diseases were Z51 (Other medical care), Q21 (Congenital malformations of cardiac septa), D61 (Other aplastic anemias), C91 (Lymphoid leukemia), J18 (Pneumonia, organism unspecified), D56 (Thalassemia), P23 (Congenital pneumonia), P07 (Disorders related to short gestation and low birth weight, not elsewhere classified), P59 (Neonatal jaundice from other and unspecified causes), P22 (Respiratory distress of newborn), K56 (Paralytic ileus and intestinal obstruction without hernia), J35 (Chronic diseases of tonsils and adenoids), D69 (Purpura and other hemorrhagic conditions), Q43 (Other congenital malformations of intestine) and P55 (Hemolytic disease of fetus and newborn). The present overall results were basically consistent across 2016, 2017 and 2018, except for No. 12--K40 (Inguinal hernia) in 2016 and No. 13-Q24 (Other congenital malformations of the heart) in 2017. Compared with the previous study (11), not all of the top diseases identified in inpatients require blood transfusion therapy. More attention should therefore be paid to establishing appropriate blood transfusion strategies and guidelines for these diseases for pediatric patients.

In addition, the most common diseases in different age-sex groups were different, as shown in Figure 2. The disease spectrum of blood transfusion in children varied greatly with age and sex. Children for 0-28 days old were mainly treated by blood transfusions for P23 (Congenital pneumonia) and P59 (Neonatal jaundice from other and unspecified causes) etc., certain conditions originating in the perinatal period. Children between 29 days and $<1$ year old were mainly treated by blood transfusions for J18 (Pneumonia, organism unspecified) and Q21 (Congenital malformations of cardiac septa), etc. Children aged 1-3 years old were mainly treated by blood transfusions for Q21 and Z51 (Other medical care), etc. Z51 and Z61 (Problems related to negative life events in childhood) were the most common diseases requiring blood transfusion in 

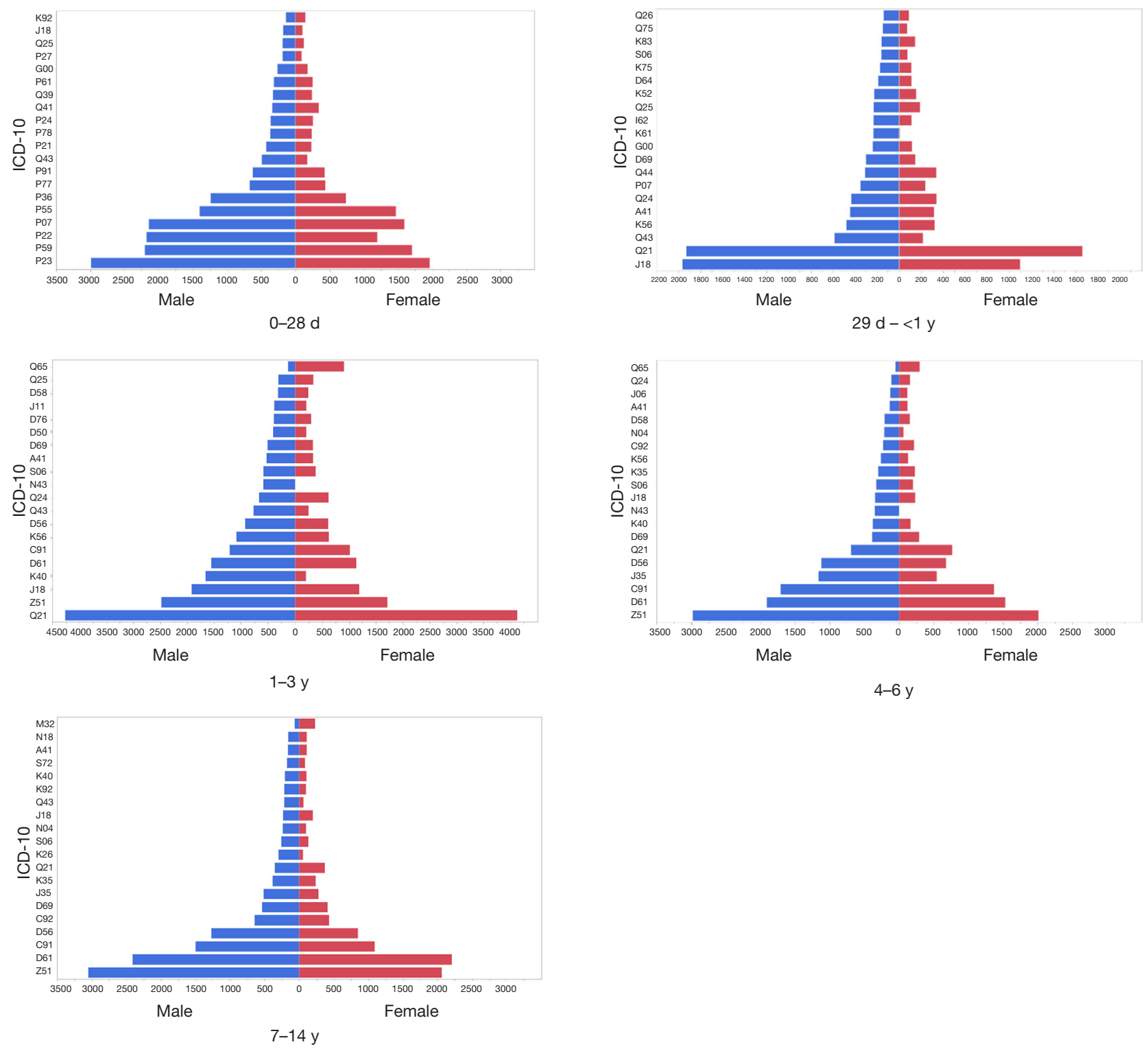

Figure 2 Population pyramid graph for the age-gender specific distribution of hospitalized children treated with blood transfusion.

the older population among the other age groups, showing that there was a greater range of transfusion diseases necessitating transfusion among children. Moreover, males had a higher proportion than females for P22 (Respiratory distress of newborn) among children aged 0-28 days old, J18 (Pneumonia, organism unspecified) and Q43 (Other congenital malformations of intestine) among children aged 29 days to $<1$ year old, K40 (Inguinal hernia) and Q43 (Other congenital malformations of intestine) among children aged 1-3 years old, and J35 (Chronic diseases of tonsils and adenoids) and N43 (Hydrocele and spermatocele) among children aged 4-6 years old. The distinct disease spectra of blood transfusion patients in different sex and age groups reflect the different characteristics of these groups, may highlight features of children's growth and development different from those of adults and indicate the need for medical attention focused on different blood transfusion strategies and guidelines.

In conclusion, this national retrospective study described and evaluated the epidemiology of blood transfusions 
stratified by sex, age, and region in an exclusively pediatric inpatient population in China. This study reveals a relatively high transfusion prevalence in the Chinese pediatric inpatient population. There are considerable differences in the prevalence and disease spectrum of transfusions between different sexes and among different ages or regions. These findings provide baseline data for establishing effective blood transfusion strategies for children in clinical practice, targeted at susceptible populations in China.

There are several limitations to this study that need to be mentioned. First, our study included 22 tertiary children's hospitals, nearly covered the major cities in Mainland China, so the data can be considered comprehensive and the results representative as a national study. However, our study still has limitations in terms of representations, especially in seven representative regions of China. Data used for this study did not include some hospitals in several provinces or regions in China, such as Hong Kong, Macao, Taiwan, Liaoning, Yunnan and Helongjiang, as data from these provinces or regions were not collected. Second, although the total number of transfusions evaluated was extremely large, the dataset was weighted toward academic medical centers and large general pediatric hospitals. Third, the use of study data in evaluating indicators associated with prevalent characteristics of blood transfusion in the pediatric inpatient population was limited.

\section{Acknowledgments}

We acknowledge investigators from members of Futang Research Center of Pediatric Development (FRCPD). We thank colleagues from Big Data and Engineering Research Center, Beijing Children's Hospital, Capital Medical University, National Center for Children's Health, Beijing, China for all their invaluable efforts.

Funding: This work was supported by the Children's Medicine Research Project of Beijing Children's Hospital, Capital Medical University (No. YZQN202003, No. YZYB202004).

\section{Footnote}

Provenance and Peer Review: This article was a standard submission to the journal. The article has undergone external peer review.

Conflicts of Interest: All authors have completed the ICMJE uniform disclosure form (available at http://dx.doi. org/10.21037/tp-20-464). The authors have no conflicts of interest to declare.

Ethical Statement: The authors are accountable for all aspects of the work in ensuring that questions related to the accuracy or integrity of any part of the work are appropriately investigated and resolved. All procedures performed in this study involving human participants were in accordance with the Declaration of Helsinki (as revised in 2013). The Ethics Committee of Beijing Children's Hospital, Capital Medical University gave expedited approval to review and use the medical data, and the requirement for informed consent was waived because of the retrospective nature of the study (2018-126).

Open Access Statement: This is an Open Access article distributed in accordance with the Creative Commons Attribution-NonCommercial-NoDerivs 4.0 International License (CC BY-NC-ND 4.0), which permits the noncommercial replication and distribution of the article with the strict proviso that no changes or edits are made and the original work is properly cited (including links to both the formal publication through the relevant DOI and the license). See: https://creativecommons.org/licenses/by-nc-nd/4.0/.

\section{References}

1. Klassen TP, Hartling L, Craig JC, et al. Children are not just small adults: the urgent need for high-quality trial evidence in children. PLoS Med 2008;5:e172.

2. New HV, Berryman J, Bolton-Maggs PH, et al. Guidelines on transfusion for fetuses, neonates and older children. $\mathrm{Br}$ J Haematol 2016;175:784-828.

3. Mueller MM, Van Remoortel H, Meybohm P, et al. Patient Blood Management: Recommendations From the 2018 Frankfurt Consensus Conference. JAMA 2019;321:983-97.

4. Vlaar APJ, Toy P, Fung M, et al. A consensus redefinition of transfusion-related acute lung injury. Transfusion 2019;59:2465-76.

5. Klein HG, Cortés-Puch I, Natanson C. Clinical practice: Blood-transfusion decisions not simple. Nature 2015;521:289.

6. Josephson CD, Luban NL. Pediatric and Neonatal Transfusion Medicine: A Roadmap for Research. Transfus Med Rev 2016;30:157-8.

7. Valentine SL, Bembea MM, Muszynski JA, et al. Consensus Recommendations for RBC Transfusion 
Practice in Critically Ill Children From the Pediatric

Critical Care Transfusion and Anemia Expertise Initiative. Pediatr Crit Care Med 2018;19:884-98.

8. Girelli G, Antoncecchi S, Casadei AM, et al.

Recommendations for transfusion therapy in neonatology. Blood Transfus 2015;13:484-97.

9. Goel R, Josephson CD, Patel EU, et al. Individual- and hospital-level correlates of red blood cell, platelet, and plasma transfusions among hospitalized children and

Cite this article as: Guo K, Wang XY, Feng GS, Tian J, Zeng YP, Ma SX, Ni X; Futang Research Center of Pediatric Development (FRCPD). The epidemiology of blood transfusion in hospitalized children: a national cross-sectional study in China. Transl Pediatr 2021;10(4):1055-1062. doi: $10.21037 /$ tp-20-464 neonates: a nationally representative study in the United States. Transfusion 2020;60:1700-12.

10. Sun Y, Ma T, Wang WH, et al. Transfusion rates and disease spectrum in neonates treated with blood transfusion in China. Medicine (Baltimore) 2020;99:e19961.

11. Feng G, Zeng Y, Tian J, et al. Disease spectrum analysis of hospitalized children in China: A study of 18 tertiary children's hospitals. Pediatr Investig 2019;3:159-64. 